\title{
Automated Quantitative Analysis of Extended Irradiation Defects - Dislocations, Voids and Precipitates in Neutron Irradiated HT-9 Steel
}

\author{
Rajat Sainju ${ }^{1}$, Colin Ophus ${ }^{2}$, Mychailo B. Toloczko ${ }^{3}$, Danny J. Edwards ${ }^{3}$ and Yuanyuan Zhu ${ }^{1,3^{*}}$ \\ 1. Department of Materials Science and Engineering, Institute of Materials Science, University of \\ Connecticut, Storrs, CT 06269, USA. \\ 2. National Center for Electron Microscopy, Molecular Foundry, Lawrence Berkeley National \\ Laboratory, Berkeley, CA 94720, USA. \\ 3. Energy and Environment Directorate, Nuclear Sciences Division, Pacific Northwest National \\ Laboratory, Richland, WA 99352, USA. \\ * Corresponding author: yuanyuan.2.zhu@uconn.edu
}

Structural alloys that make up the core components of nuclear reactors are exposed to radiation that can substantially alter the microstructure and mechanical properties of the material. Irradiation can cause the formation of extended defects including dislocation line and loops, voids and various types of precipitates. To understand the irradiation effects, it is critical to characterize these defects individually and collectively to quantify how they evolve throughout irradiation stages. Transmission electron microscopy (TEM) is a standard tool for providing extensive size, number density and spatial distribution of extended irradiation defects, however, manual measurement of defect size and number density requires time-intensive effort to analyze enough defects that provide a statistically meaningful result and are prone to errors of subjectivity. In this work, we developed a set of MATLAB algorithms offering robust and automated identification and quantification of extended irradiation defects like dislocation lines, voids and precipitates commonly observed in neutron irradiated cladding alloys [1].

Using a neutron-irradiated HT-9 martensitic alloy irradiated at $412^{\circ} \mathrm{C}$ to $111.8 \mathrm{dpa}$, we demonstrate the capability of these algorithms for quantitative defect analysis. Figure 1 presents defect images of the HT9 using an advanced diffraction contrast imaging scanning transmission electron microscopy (DCI STEM) that suppresses bend contours and provides better defect contrast compared to conventional TEM [2]. The first step of defect analysis is defect identification, a process that can be digitally achieved by contrast thresholding (for simple defect contrast) and by machine learning computer vision for defect semantic segmentation [3]. Here, labeled binary defect images (Figures 2a and 2c) were used. For dislocation density quantification, we employed an optimized circular-grid intersection method first introduced by Dr. John Steeds [4]. This method is shown in Figures 2a and 2b, where the number of intersections of dislocation lines with circular traces was measured. This produces an unbiased sampling of dislocation lines, regardless of their orientation. This process was optimized with automated digital intersection number and grid length registration. Interestingly, it was found that a relatively large number of circles in a set circular gird is was needed to produce an invariant result (Figure 2b), with a low systematic measurement error. This demonstrates the main advantage of computer-based defect analysis, in performing repetitive operations quickly with a reduced systematic error. For the quantitative analysis of voids and precipitates a challenge encountered with automated defect analysis is feature overlap, particularly when the density of voids/precipitates is high. To mitigate this problem, as shown in Figure 2d, we modified the popular circular Hough transformation and treated 'circular shape' as the particle descriptor for voids/precipitates, efficiently resolving the overlapping particles. In summary, this automated digital quantification together with machine learning enabled defect identification [3] opens 
new opportunities for standardizing the characterization workflow for quantitative analysis of extended irradiation defects.

\section{References:}

[1] R Sainju, C Ophus, MB Toloczko and DJ Edwards and Y Zhu, in preparation

[2] Y Zhu, C Ophus, MB Toloczko and DJ Edwards, Ultramicroscopy, 193 (2018) 12-23.

[3] G Roberts, SY Haile, R Sainju, D Patel, DJ Edwards, B Hutchinson and Y Zhu. in preparation

[4] JW Steeds, Proceedings of the Royal Society A, 292 (1966), 343-373.

[5] The authors acknowledge funding from the U.S. Department of Energy Office of Nuclear Energy's Nuclear Energy Enabling Technologies program project CFA 16-10570, Office of Fusion Energy

Sciences under contract DE-AC05-76RL01830, and for Molecular Foundry which is supported by the Office of Basic Energy Sciences, under Contract No. DE-AC02-05CH11231.
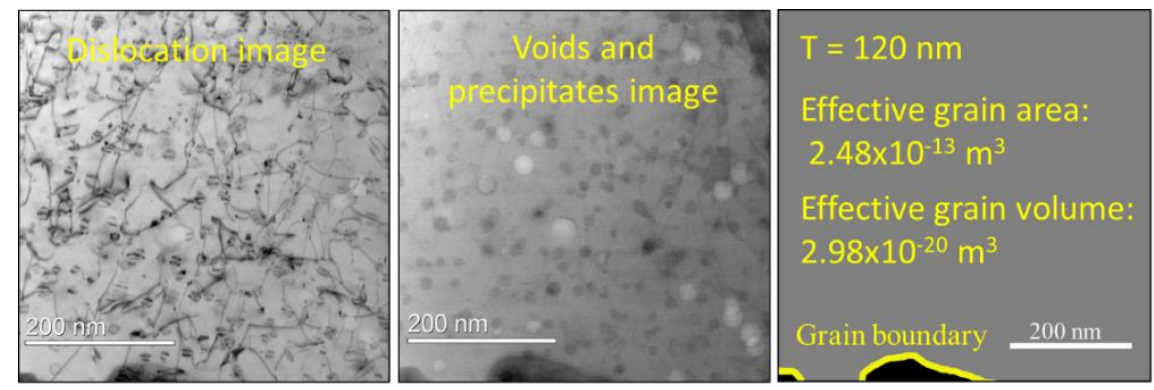

Figure 1. DCI STEM imaging of (left) dislocations, (center) voids and precipitates, and (right) effective grain area and volume calculated by the algorithm in a sample with a thickness (T) of $120 \mathrm{~nm}$.
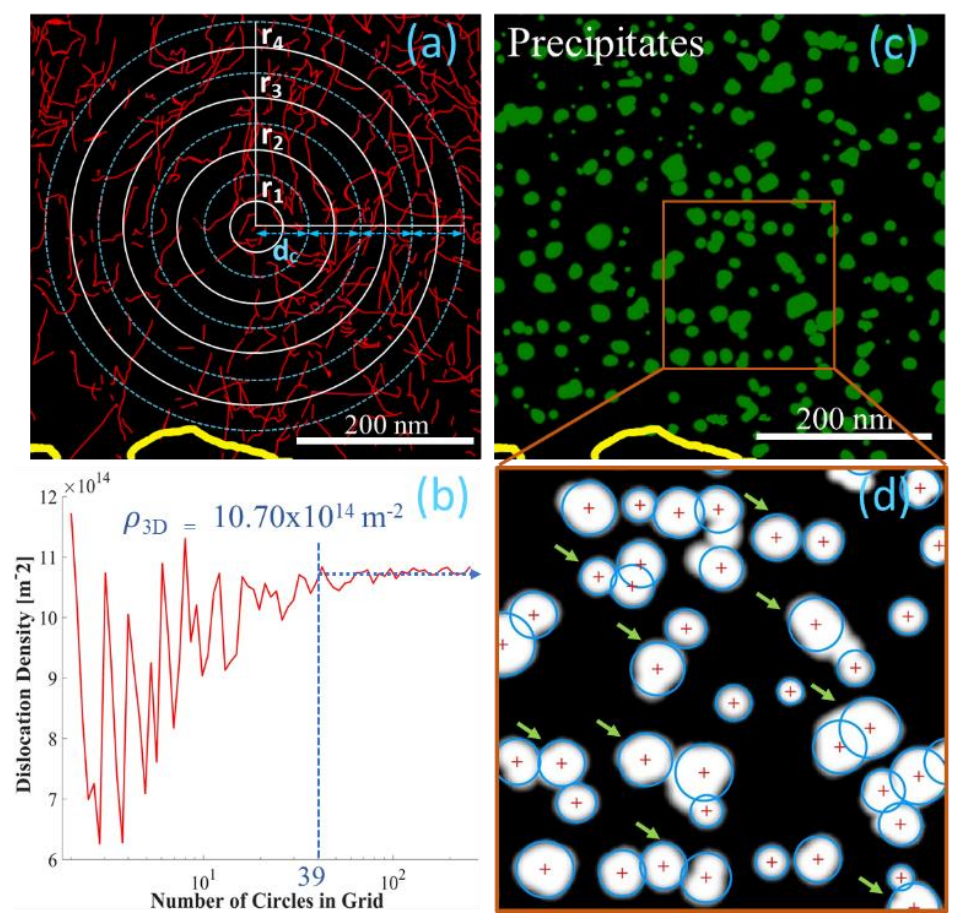

Figure 2. An illustration of the algorithm used for quantification of (a) and (b) dislocation density using the optimized circular intersection method with recommended threshold for the number of circles, (c) and (d) size and density of precipitates by modified circular Hough transformation to overcome overlapping. 